vaste champ d'exploration aux linguistes et aux stylisticiens, non seulement sur l'œuvre mais sur toute la période ; richesse de la contextualisation historique et littéraire fournie dans l'introduction et les notes ; intérêt des annexes (tableaux, dossier iconographique, index multiples). Cette réédition dans une collection financièrement accessible met à la portée de tous les chercheurs un travail scientifique de premier plan dont on a pu apprécier depuis longtemps la rigueur et l'exigence, et où le discernement le dispute au souci d'authenticité. On pourra évidemment revenir sur tel ou tel détail (dans la Préface, ne peut-on voir une coquille dans le v. 320 où Tu feras rire l'assistance serait sans doute plus satisfaisant que Te feras rire ?), ou regretter que l'opulente bibliographie n'ait pas été remise à jour, mais le lecteur pourra toujours se reporter aux mises à jour régulières de la bibliographie albinéenne qui paraissent dans la revue Albineana, Cahiers d'Aubigné, également diffusée par les éditions Champion. Il faut souhaiter que se poursuive cette politique de diffusion des grandes éditions de grands textes dans une collection qui met à la portée de chacun un matériau de premier ordre, indispensable à la recherche en littérature et en langue du Xvi ${ }^{\mathrm{e}}$ siècle.

MARIE-HÉLÈNE PRAT-SERVET, Université Jean Moulin-Lyon 3

\title{
Servir Dieu, le Roi et l'Etat. Philippe Duplessis-Mornay (1549-1623)
}

Actes du colloque de Saumur (13-15 mai 2004), réunis par Hugues Daussy et Véronique Ferrer, Albineana 18 (2006), 696 p.

Ce très riche volume propose trente-deux communications qui couvrent les différentes facettes de l'œuvre et de la vie de Ph. Duplessis-Mornay. On y lira des études dues aux plus grands spécialistes de cette figure centrale de l'histoire de France et de la pensée religieuse. Quatre grandes parties le structurent.

La première partie s'attache à la théologie et à la controverse religieuse. M.-M. Fragonard retrouve dans le Traité de l'Eglise un imaginaire marqué par la violence. D. Ménager examine le traité De la Verité de la Religion chrestienne et y analyse l'approche du Judaïsme tandis que M. Yardéni examine la place des Juifs dans les écrits polémiques et théologiques ; elle y voit avant tout les traces de la mentalité française du temps. B. Roussel suit la trace d'un Duplessis-Mornay exégète biblique ; il dévoile un auteur qui n'élabore pas une herméneutique élaborée, mais montre que tous peuvent être théologiens. M. Holt examine la place du sacrement de la Cène que Duplessis-Mornay expose en termes sociaux. Plus proche des stratégies d'écriture, C. Huchard s'interroge sur l'art de résoudre les controverses sous sa 
plume et examine des «stratégies polémiques et politiques » d'auteur ; comme en prolongement de ces analyses, G. Schrenck lit un ouvrage de Jean de Bordes, les Etcaetera de Duplessis-Mornay, qui s'inscrit dans les mêmes controverses. La section se clôt par une contribution de $\mathrm{B}$. Conconi qui s'intéresse à la réception italienne des écrits de Duplessis-Mornay marquée par la traduction de ses ouvrages de théologie.

La deuxième partie porte sur « littérature et spiritualité ». M. Engammare examine les spécificités théologiques des Méditations sur les Proverbes, P. Blum-Cuny les formes du bonheur qu'envisagent les Méditations sur les psaumes; ces dernières Méditations sont lues par V. Ferrer qui dévoile les ressorts de la «vraye voix de l’ame ». M.-D. Legrand trouve une illustration de la morale calvinienne dans les Larmes. M. Richter lit quelques poèmes où s'inscrit la ferveur politique et religieuse de l'auteur et G. Banderier établit un parallèle, toujours autour de l'écriture poétique, entre Duplessis-Mornay et Du Bartas.

La troisième partie est consacrée au « combat politique ». P.-A. Mellet examine les liens étroits qui unissent droit divin, naturel et civil autour de la lecture que Duplessis-Mornay propose du chapitre XIII de l'épître de saint Paul aux Romains. Pour J. Collins, on voit dans l'action de Duplessis-Mornay les traces d'une évolution qui conduit de la « République française à l'État français ». Dans le domaine religieux, F. Chevalier analyse son action dans le cadre des synodes nationaux et en dégage sa volonté de restaurer l'union des Églises réformées. N. Fornerod examine le complexe thème du concile qui parcourt l'œuvre, mais aussi la vie du gouverneur de Saumur. A. Bettoni présente et analyse un document des archives de Padoue qui lui permet de s'attacher aux relations qui unirent Duplessis-Mornay à Du Ferrier, ambassadeur de France à Venise ; elle souligne la place des liens de familiarité qui soudent les groupes et donne un sens particulier au service du roi. A. Denis examine la position de Venise qui prend ses distances face à Rome au début du XVII ${ }^{\mathrm{e}}$ siècle et la place que Duplessis-Mornay occupe dans les souhaits de faire triompher la Réforme dans la Sérénissime. Cette dimension internationale de l'action de Duplessis-Mornay se retrouve avec le long article que M. Greengrass consacre à l'idéal qu'il représente en Angleterre sous le règne de Jacques $1^{\mathrm{er}}$ tant dans les domaines religieux que politiques; la liste des traductions anglaises de ses œuvres entre 1576 et 1623 témoigne du rayonnement de sa pensée. Sous le titre «Géopolitique de Duplessis-Mornay », F. Lestringant épouse le regard évolutif que le calviniste porte sur les continents autres que l'Europe, considérés comme lieux d'une éventuelle solution aux problèmes religieux, politiques et économiques européens. D. Poton examine la position de Duplessis-Mornay entre 1617 et 1621, 
période au cours de laquelle tentent de s'équilibrer le service d'un roi en guerre contre les Huguenots et la défense de l'Eglise. Dans un registre assez différent, E. Surget parcourt quant à lui la géographie de l'espace qui structure l'imaginaire du gouverneur de Saumur.

La dernière partie se place sous le signe de l'intime amical, familial et des activités civiques. Contre-exemple tout d'abord avec la relation avec Sully: F. Barbiche présente deux hommes certes unis par la foi, mais dont les visions profondes étaient inconciliables. B. Nicollier relit la correspondance avec Th. de Bèze et souligne les convergences de vue tout en remarquant que l'exclusif service de Dieu de Bèze ne répondait pas aux aspirations de Duplessis-Mornay. Véritable amitié par contre avec Philippe Sydney que R. Kuin retrace. Selon N. Kuperty-Tsur, la figure qui émerge des Mémoires de Charlotte Duplessis-Mornay, femme de Philippe, tient de l'apologie autant que de l'hagiographie destinée au fils. Toujours à la lecture de ces Mémoires, l'article que signent S. Broomhall et C. Winn examine cette figure féminine prise dans les liens des devoirs conjugaux et familiaux tandis que D. Davis Donald la considère comme mère et médiatrice familiale. Les pages de J. Rosenthal, « L'affaire de la coiffure », permettent de comprendre comment les voix du mari et de la femme se complètent dans un cadre théologique. La dernière communication change radicalement de domaine ; E. Cron examine le rôle actif de Duplessis-Mornay dans la politique de construction urbaine de Saumur.

Les conclusions d'O. Millet ne se veulent pas une synthèse ; elles permettent néanmoins de saisir les liens étroits qui unissent les différentes communications et soulignent ainsi la cohérence d'une figure aux multiples facettes. Une bibliographie des travaux consacrés à Duplessis-Mornay et un index complètent le volume. Celui-ci précise certains points et ouvre de nombreuses perspectives; il est par-là une étape essentielle aux études consacrées aux grandes figures autour desquelles se cristallisent les changements qui conduisent de la Renaissance au XVII ${ }^{\mathrm{e}}$ siècle.

BRUNO PETEY-GIRARD, Université de Paris XII 